\title{
Note
}

\section{Influence du régime antérieur sur la quantité de fourrage ingérée par des moutons mâles castrés}

\author{
F. GATEL et Brigitte MICHALET-DOREAU \\ avec la collaboration technique de L. L'Hotelier \\ I.N.R.A., C.R.Z.V. de Theix, Laboratoire des Aliments \\ Centre de Recherches de Clermont-Ferrand, F 63122 Ceyrat
}

\begin{abstract}
Résumé
Pour étudier l'influence du régime antérieur sur la quantité de fourrage ingérée par des moutons, six béliers castrés ont d'abord reçu pendant cinq semaines des régimes correspondant à des niveaux alimentaires très différents ( 80 à 180 p. 100 des besoins d'entretien), puis un même foin distribué à volonté ; nous avons mesuré durant cette dernière période les quantités de foin ingérées ainsi que le poids vif et l'état d'engraissement des animaux. Nous n'avons pas observé d'influence du régime précédemment distribué sur les quantités de foin ingérées lors de la période de mesure.
\end{abstract}

Mots clés : Quantités ingérées, état d'engraissement, succession de régimes, moutons.

\section{Introduction}

Dans le nouveau système des Unités d'Encombrement proposé par l'I.N.R.A. (JARRIGE et al., 1980), l'ingestibilité des fourrages est déterminée à partir de la quantité journalière de matière sèche, exprimée en $\mathrm{g} / \mathrm{kg} \mathrm{P}^{0,75}$, ingérée par des moutons «standards» (béliers castrés, de race Texel, âgés de 1 à 4 ans, et pesant environ $70 \mathrm{~kg}$ ) durant une semaine, après deux semaines de mise en régime. Cela suppose que la capacité d'ingestion de ces animaux soit à peu près constante et notamment que les quantités ingérées d'un fourrage donné dépendent peu ou pas du régime distribué pendant la période de mesure précédente ou des variations d'état d'engraissement des animaux qui pourraient en résulter ; c'est ce que nous avons voulu vérifier en mesurant avec un même foin les quantités ingérées par des animaux ayant reçu précédemment des régimes alimentaires très différents.

\section{Matériel et méthodes}

Six béliers castrés, de race Texel, âgés de quatre ans, et pesant en moyenne $83,0 \pm 4,9 \mathrm{~kg}$ ont été placés en case individuelle et alimentés avec du foin de Dactyle 
(deuxième cycle, âgé de 11 semaines) distribué seul ou avec du concentré. Les caractéristiques de ce foin étaient les suivantes : matière sèche : 89,8 p. 100 ; matières organiques : 91,4 p. $100 \mathrm{MS}$; matières azotées : 11,1 p. $100 \mathrm{MS}$; cellulose brute : 25,8 p. $100 \mathrm{MS}$; digestibilité de la matière organique : 67,4 p. 100.

\section{TABLeaU 1}

Régimes alimentaires.

Diets.

\begin{tabular}{|c|c|c|}
\hline & $\begin{array}{l}\text { Régime } \\
\text { Diet }\end{array}$ & $\begin{array}{l}\text { Niveau d'alimentation } \\
\text { Feeding level }\end{array}$ \\
\hline B & $\begin{array}{l}\text { Foin distribué à raison de } 50 \% \text { de la quantité ingérée } \\
\text { à volonté - Hay fed in restricted amount (50\% of } \\
\text { voluntary intake) }\end{array}$ & $\begin{array}{c}0,8 \\
(0,74 \stackrel{-0,97)}{ }\end{array}$ \\
\hline $\mathbf{M}$ & $\begin{array}{l}\text { Foin distribué à raison de } 90 \% \text { de la quantité ingérée à } \\
\text { volonté - Hay fed in restricted amount }(90 \% \text { of voluntary } \\
\text { intake) }\end{array}$ & $\left(1,17^{1,3}-1,52\right)$ \\
\hline $\mathbf{H}$ & $\begin{array}{l}\text { Mélange foin }(70 \%) \text { et concentré }(*)(30 \%) \text { distribué à } \\
\text { volonté - Hay }(70 \%) \text { and concentrate }(30 \%) \text { fed ad } \\
\text { libitum }\end{array}$ & $\begin{array}{c}1,8 \\
(1,60-1,90)\end{array}$ \\
\hline
\end{tabular}

(*) Composition du concentré : orge $=20$ p. 100 ; maïs $=20$ p. 100 ; pulpe $=30$ p. $100 ;$ tourteau de soja $50=12$ p. $100 ;$ tourteau de colza $=10$ p. 100 ; mélasse $=5$ p. 100 .

Composition of the concentrate : barley : 20 p. 100 ; maize : 20 p. 100 ; pulp : 30 p. 100; soyabean oil-meal : 22 p. 100 ; molasses : 5 p. 100.

Nous avons utilisé trois régimes (tabl. 1) correspondant à des niveaux alimentaires bas (B), moyen $(\mathrm{M})$ ou haut $(\mathrm{H})$. Ces régimes étaient distribués aux animaux pendant une période de cinq semaines («période préexpérimentale») à la suite de quoi les animaux recevaient le foin seul à volonté (10 p. 100 de refus) pendant trois semaines ( "période de mesure»). Les six moutons ont été répartis en trois lots, chaque lot de deux moutons recevant successivement chacun des trois régimes selon un dispositif en carré latịn.

Durant chaque «période de mesure», nous avons mesuré pour chaque animal les quantités ingérées par jour, le poids vif, et au cours de la seconde semaine, l'état d'engraissement (méthode de l'espace de diffusion de l'eau lourde, RobELIN, 1973 ; TISSIER et al., 1978 ; TISSIER et al., 1983). 


\section{Résultats et discussion}

Pendant la période où $\mathrm{le}$ foin est distribué seul à volonté (périodes de mesure), les quantités ingérées sont respectivement de $59,9,56,3$ et $55,7 \mathrm{~g} / \mathrm{kg} \mathrm{P}$ 0,75 (différences non significatives) après les régimes $B, M$ et $H$.

Ces régimes correspondent à des niveaux alimentaires extrêmes pour nos animaux (respectivement 80,130 et 180 p. 100 des besoins théoriques d'entretien). Les quantités ingérées pendant les "périodes de mesure » sont donc indépendantes du niveau alimentaire appliqué lors des périodes préexpérimentales précédentes.

L'état d'engraissement des animaux (quantité de lipides corporels, exprimés en p. 100 du poids vif) déterminé au cours de la «période de mesure » est respectivement de $31,3,31,0$ et 29,8 p. 100 (différences non significatives) après les régimes $B$, $M$ et H. Donnely, Davidson, Freer (1974) ont obtenu des moutons présentant des états dengraissement différents au bout de 7 semaines, mais leurs animaux se trouvaient encore en croissance (33 kg au départ). Par contre, avec des moutons adultes (50 kg au départ), Graham (1969) a dû maintenir un régime préexpérimental pendant 15 mois pour avoir de fortes variations d'état d'engraissement (13 à $33 \mathrm{p}$. 100). Il n'est donc pas surprenant que la distribution, pendant 5 semaines, de régimes alimentaires très différents, n'ait pas modifié l'état d'engraissement de nos animaux, adultes, et de surcroît assez gras (30,7 p. 100).

Enfin, il n’y a pas de liaison entre les quantités ingérées, exprimées en $\mathrm{g} / \mathrm{kg} \mathrm{P}^{0,75}$ et l'état d'engraissement des animaux lorsque l'on prend en compte tous les individus lors de toutes les périodes $(\mathrm{n}=18, \mathrm{r}=0,42)$. Donnely, Davidson, Freer (1974) étaient arrivés aux mêmes conclusions avec des animaux plus maigres dont l'état d'engraissement variait de 11 à 18 p. 100. Cependant, la capacité d'ingestion peut être liée à l'état d'engraissement quand ce dernier varie de façon très importante, comme cela a été montré sur des béliers castrés (Graham, 1969) ou sur des femelles taries non gravides (Bines, Suzuki, Balch, 1969, sur vaches; FOOT, 1972, sur brebis) : les plages de variation d'état d'engraissement ou de poids vif étaient respectivement de 13 à 33 p. 100 pour les béliers, 430 à $610 \mathrm{~kg}$ pour les vaches, 47 à $72 \mathrm{~kg}$ pour les brebis, alors que dans notre essai, l'état d'engraissement variait de 26 à 34 p. 100 et le poids vif de 79 à $97 \mathrm{~kg}$.

\section{Conclusion}

Chez des animaux âgés et relativement gras, nous n’observons donc pas d'influence du régime distribué au cours d'une période sur la capacité d'ingestion pendant la période de mesure suivante; mais il reste possible qu'une succession de régimes de valeur alimentaire faible ou élevée puisse entraîner progressivement des modifications de plus en plus importantes de l'état d'engraissement de nos animaux et par là, faire varier la capacité d'ingestion. 


\section{Summary}

Effect of previous diet on feed intake by wethers

In order to study the effect of previous diet on feed intake by sheep, six wethers were fed various diets supplying 80 to 180 per cent of maintenance requirement (table 1) for five weeks, then cocks-foot hay ad libitum. During the latter period daily feed intake was recorded, and live weight and fatness of sheep were measured. Daily hay intake did not differ significantly between animals fed one or the other diet, neither was there any differences in fatness. The results suggest that five weeks are not long enough to markedly affect the fatness of adult and already fat sheep, and the diet given during one short experiment has no residual effect on feed intake in the following experiment.

Key words : Feed intake, fatness, succession of diet, sheep.

Reçu en mai 1983.

Accepté en août 1983.

\section{Références bibliographiques}

Bines J.A., Suzuki S., Balch C.C., 1969. The quantitative significances of long terme regulation of food intake in the cow. Br. J. Nutr., 23, 695-704.

Donnely J.R., Davidson J.L., Freer M., 1974. Effect of body composition on the intake of food by mature sheep. Aust. J. Agric. Res., 25, 813-823.

FooT J.Z., 1972. A note on the effect of body composition on the voluntary intake of dried grass wafers by scottish blackface ewes. Anim. Prod., 14, 131-134.

Graham N. MC C., 1969. The influence of body weight (fatness) on the energetic efficiency of adult sheep. Aust. J. Agric. Res., 20, 375-385.

Jarrige R., Beranger C., Demarquilly C., Dulphy J.P., Geay Y., Hoden A., Journet M., Malterre C., Micol D., Petrt M., Robelin J., 1980. Le système des Unités d'Encombrement pour les bovins. in «Alimentation des Ruminants », 599-621. Ed. I.N.R.A., Versailles.

Robelin J., 1973. Estimation de la composition corporelle des animaux à partir des espaces de diffusion de l'eau marquée. Ann. Biol. anim. Biochim. Biophys., 13, 285-305.

Tissier M., Robelin J., Purroy A., Geay Y., 1978. Extraction et dosage automatique de l'eau lourde dans les liquides biologiques. Ann. Biol. anim. Biochin. Biophys., 18, 1223-1228.

Tissier M., Theriez M., Purroy A., Bocquier F., 1983. Estimation in vivo de la composition corporelle de la brebis par la mesure de l'espace de diffusion de l'eau lourde. Reprod. Nutr. Develop., 23, 693-707. 